\title{
Isolation, Screening and Characterization of Zinc Solubilizing Microorganisms from Direct Sown Paddy (Oryza sativa L.) Rhizospheric Soils of Andhra Pradesh
}

\author{
S. Vinod Babu ${ }^{*}$, A. Vijaya Gopal ${ }^{1}$, N. Trimurtulu ${ }^{1}$, \\ G. Kishore Babu ${ }^{2}$ and B. Sree Lakshmi ${ }^{3}$ \\ ${ }^{1}$ Department of Agricultural Microbiology, Advanced Post Graduate centre, \\ ANGRAU, Lam, Guntur, India \\ ${ }^{2}$ Department of Soil Science, ${ }^{3}$ Department of Plant Pathology, RARS, \\ ANGRAU, Lam, Guntur, India \\ *Corresponding author
}

\section{A B S T R A C T}

\section{Keywords \\ Rice (Oryza sativa L.), Rhizosphere, Zinc Solubilizing Bacteria (ZnSB) and Zinc phosphate $\left[\mathrm{Zn}_{3}\left(\mathrm{PO}_{4}\right)_{2}\right]$.}

\section{Article Info}

Accepted:

24 August 2020

Available Online:

10 September 2020
Rice (Oryza sativa L.) is one of the vital staple food for more than $50 \%$ of the world's population provided that major source of the food energy. Rice occupies a key role in Indian agriculture contributing to $20-25 \%$ of agricultural income. It pays about $43 \%$ of the whole food grain production and about $46 \%$ of the overall cereal production in India (FAO, 2017).In the present study collected twenty (20) direct sown paddy rhizosphere soil samples from four districts of Andhra Pradesh and recorded their geographical position of collected soil samples. From that thirty two (32) Zinc Solubilizing Bacteria (ZnSB) isolated by using zinc phosphate $\left[\mathrm{Zn}_{3}\left(\mathrm{PO}_{4}\right)_{2}\right]$ as a insoluble source of zinc in the trismineral salts media. Zinc solubilizing isolates coded based on their soil sample collected geographical position, recorded the morphological, cultural characteristics of $\mathrm{ZnSB}$. ZnSB Isolates screened based on their zinc solubilization efficiency both qualitatively and quantitatively. Among all $32 \mathrm{ZnSB}$ isolates $\mathrm{Zn} \mathrm{KJJ}-4$ has the high ability to solubilize zinc both qualitatively and quantitatively among the all and lowest was showed by ZnAUU-1. Zn KJJ-4 showed the positive for the starch hydrolysis test, hydrogen sulphide test, catalase test, oxidase test, gelatine liquefaction test, methyl red test, Vogues Proskauer test, citrate utilization test and ammonia production test. ZnKJJ-4 showed the negative for indole production test.

\section{Introduction}

The plants essential several macro and micro nutrients for their growth and development. Zinc ( $\mathrm{Zn})$ is one of the most important micronutrients is necessary for the normal healthy growth and reproduction of plants. In rice zinc has the crucial role for the growth and development. In the recent days importance of Zinc Solubilizing Bacteria (ZSB) has increased and these are probable candidate for improving bioavailable fraction of $\mathrm{Zn}$ to host plant for enlightening the crop growth, yield and quality without affecting the environment is being adopted in the agriculture. Alternatively, several 
microorganisms, especially those associated with roots, have the ability to rise plant growth and productivity (Rodriguiz et al., 2004) by enhancing the supply of mineral nutrients of low mobility in the soil like $\mathrm{P}, \mathrm{Zn}$ and $\mathrm{Cu}$ (Thompson, 1996). Bacterial strains that have been reported to show zinc solubilisation on lab scale include Pseudomonas aeruginosa (Fasim et al., 2002), Gluconacetobacter diazotrophicus (Saravanan et al., 2007), Bacillus spp., Pseudomonas striata, Pseudomonas fluorescence, Burkholderia cenocepacia (Pawar et al., 2015), Serratia liquefaciens, Serratia marcescens, and Bacillus thuringiensis (Abaid - Ullah et al., 2015).

\section{Materials and Methods}

\section{Collection of soil samples}

Rhizospheric soils were collected from different places of Kurnool, Prakasam, Guntur and Anantapur districts such as soils of direct sown paddy growing areas by using quadratic method of soil sample collecting procedure. In Kurnool District (Atmakur, Kothapalle, Jupadu bungalow, Pamulapadu and Velugodu), Prakasam District (Tripuranthakam, Yerragondapalem, Dornala, Markapuram and Giddalur), Guntur District (Vinukonda, Narasaropet, Chilakaluripet, Sattenapalle and Piduguralla) and Anantapur District (Guntakal, Gooty, Pamidi, Tadipatri and Uravakonda) along with particular GPS coordinates for each sampling area was fixed (Table 1) soil samples were collected from each Mandal from different farmer's fields.

Isolation of different bacterial isolates following serial dilution method and plating techniques using suitable media and incubated conditions were followed. Pure cultures must be obtained by the streak plate method. These pure cultures of different bacterial isolates were preserved and used for further analysis.
Characterization of zinc solubilizing bacterial isolates by morphological, cultural and biochemical characters

\section{Morphological characterization}

All the zinc solubilizing bacterial isolates were checked for their purity and then studied for the colony morphology and pigmentation. The cell shape and gram reaction was also recorded as per the standard procedures given by Barthalomew and Mittewar (1950).

\section{Colony morphology}

Morphological characteristics of the colony of each isolate were examined on Nutrient agar and specialized medium and incubated for according to isolate. Cultural characterization of isolates was observed by different characteristics of colonies such as shape, size, elevation, surface, margin, colour, odour, pigmentation etc. were recorded.

\section{Biochemical and physiological characterization of zinc solubilizing bacterial isolates}

\section{Starch hydrolysis (MacFaddin, 2000)}

Sterile starch agar plates were spotted with 10 $\mu l$ overnight broth cultures of the isolates and incubated at $28 \pm 2{ }^{\circ} \mathrm{C}$ for $24-48$ hours. After incubation, the plates were flooded with an iodine solution. The formation of a transparent zone around the colony was taken as a positive reaction to the test.

\section{Hydrogen sulfide test (Beishir, 1991)}

Sterilized Hydrogen Sulfide-Indole-Motility agar (SIM agar) stabs were inoculated along the wall of the tubes with overnight cultures of the isolates and incubated for 48 hours at $28 \pm 2{ }^{\circ} \mathrm{C}$. Visualization of black colour along the line of inoculation indicated a positive reaction to the test. 
Indole production (Isenberg and Sundheim, 1958)

Sterilized hydrogen sulfide-indole-motility agar (SIM agar) slants were inoculated with the overnight cultures of the isolates and incubated for 48 hours at $28 \pm 2^{\circ} \mathrm{C}$. Following incubation, 10 drops of Kovac's indole reagent was added to each tube. The isolates showing the production of red colour were recorded as positive for indole production.

\section{Catalase test (Rangaswami and Bagyaraj, 1993)}

This test was performed to study the presence of catalase enzyme in bacterial colonies. Pure isolates (24 hours old) were taken on glass slides and one drop of $\mathrm{H}_{2} \mathrm{O}_{2}(30 \%)$ was added. The appearance of the gas bubble indicated the presence of catalase enzyme.

\section{Oxidase test (Collins and Lyne, 1970)}

The overnight cultures of the test isolate were spotted on plates poured with sterile Trypticase Soy Agar (TSA) and the plates were incubated for 24 hours at $28 \pm 2^{\circ} \mathrm{C}$. After incubation, 2-3 drops of $\mathrm{N}, \mathrm{N}, \mathrm{N}, \mathrm{N}$ tetramethyl-p-phenylenediamine-dihydro chloride (Wurster's reagent) was added onto the surface of growth of each test organism. The isolates showing the change of colour to maroon were noted as oxidase positive.

\section{Gelatin liquefaction (MacFaddin, 2000)}

The overnight cultures of the test isolates were inoculated to sterilized nutrient gelatin deep tubes and incubated for 24 hours at $28 \pm$ $2^{\circ} \mathrm{C}$. Then the tubes were kept in the refrigerator for 30 minutes at $4^{\circ} \mathrm{C}$. The isolates showing liquefied gelatin were taken as positive and those which resulted in the solidification of gelatin on refrigeration were recorded as negative for the test.

\section{Methyl red test (Crown and Gen, 1998)}

Sterilized glucose-phosphate broth tubes were inoculated with the test culture and incubated at $28 \pm 2^{\circ} \mathrm{C}$ for 48 hours. After incubation five drops of methyl red indicator were added to each tube and gently shaken. Red colour production was taken as positive and yellow colour production was taken as negative for the test.

\section{Vogesprausker's test (MacFaddin, 2000)}

To the pre-sterilized glucose-phosphate broth tubes, test cultures were inoculated and incubated at $37^{\circ} \mathrm{C}$ for 48 hours. After incubation ten drops of Barritt's reagent-A was added and gently shaken followed by the addition of 10 drops of Barritt's reagent-B. The development of pink colour in the broth was taken as positive for the test.

\section{Citrate utilization (MacFaddin, 2000)}

Isolates were streaked on Simmon's citrate agar slants and incubated at $28 \pm 2^{\circ} \mathrm{C}$ for 24 hours. Change in colour from green to blue indicates the positive reaction for citrate utilization.

\section{Ammonia production (Juanda, 2005)}

The isolates were tested for ammonia production by inoculating the isolates into 10 $\mathrm{ml}$ of pre-sterilized peptone water in test tubes. The tubes were incubated for 48-72 hours at $36 \pm 2^{\circ} \mathrm{C}$. After that Nessler's reagent $(0.5 \mathrm{ml})$ was added in each tube. Change in colour of the medium from brown to yellow colour was taken as a positive test for ammonia production.

\section{Isolation of zinc solubilizing bacteria}

Serial dilution method was used for isolating the Zinc Solubilizing Bacteria $(\mathrm{ZnSB})$ from the rhizosphere soil samples. $1.0 \mathrm{~g}$ of rice rhizosphere samples was suspended in $9.0 \mathrm{ml}$ 
of saline distilled water in test tubes. From the first dilution $1 \mathrm{ml}$ was transferred to test tube containing $9 \mathrm{ml}$ of sterile distilled water to get $10^{-2}$ dilution. The same method was followed for preparing up to $10^{-6}$ dilution. The trismineral salts medium was prepared containing dextrose - $10.0 \mathrm{~g},\left(\mathrm{NH}_{4}\right)_{2} \mathrm{SO}_{4}-1.0$ g, $\mathrm{KCl}-0.2 \mathrm{~g}, \mathrm{~K}_{2} \mathrm{HPO}_{4}-0.1 \mathrm{~g}, \mathrm{MgSO}_{4}-0.2 \mathrm{~g}$ in distilled water $(1000 \mathrm{ml}$ and $\mathrm{pH}$ was adjusted 7.0). The source of insoluble zinc compounds such as $\mathrm{ZnO}, \mathrm{ZnCO}_{3}$ and $\mathrm{Zn}_{3}$ $\left(\mathrm{PO}_{4}\right)_{2}$ was supplemented at $1 \%$ and agar was added into the medium individually and autoclaved at $121^{\circ} \mathrm{C}$ for $30 \mathrm{~min}$. The agar medium was poured in sterile petri plates under aseptic condition. After solidification $0.1 \mathrm{ml}$ from $10^{-4}, 10^{-5}$ and $10^{-6}$ dilutions of rice rhizosphere soil samples were taken by sterile pipette, transferred and spread on to petri plates. The inoculated plates were incubated at $27-30^{\circ} \mathrm{C}$ for $48 \mathrm{~h}$.

\section{Qualitative and quantitative assay for zinc solubilization}

Zinc solubilizing ability of bacterial isolates were evaluated using zinc phosphate in both plate and broth media assays.

\section{Plate assay (Qualitative study)}

All the bacterial strains were screened for their ability to solubilize zinc in mineral salts agar medium was amended with $0.1 \%$ of zinc phosphate $\mathrm{Zn}_{3}\left(\mathrm{PO}_{4}\right)_{2}$. The actively growing cultures $(5 \mu \mathrm{l})$ were spot inoculated onto the medium, incubated at $28^{\circ} \mathrm{C}$ and solubilization zone will be measured after 5 days of inoculation and cleared zone was expressed as solubilization efficiency in percent and area in square millimeter $(\mathrm{mm})$.

\section{Zinc solubilization in broth assay (Quantitative study)}

Analysis of insoluble zinc solubilized in liquid medium by the bacterial culture was determined quantitatively by following the protocol of Fasim et al., (2002) using Atomic Absorption Spectrophotometer (AAS). In the broth assay, the selected bacterial isolates were grown in $100 \mathrm{ml}$ of mineral salts medium broth supplemented with insoluble source of zinc phosphate at $0.1 \%$ concentration and incubated for $48 \mathrm{hrs}$ at $28^{\circ} \mathrm{C}$ in an orbital shaker at $120 \mathrm{rpm}$. There were three replicates $(n=3$ for each treatment) maintained for each bacterial isolates. The cultured broth was filtered through Whatman No. 42 filter paper and centrifuge at 10,000 rpm for 10 minutes. The culture supernatant was fed directly to the Atomic Absorption Spectroscopy (Varian Model SPECTRAA 220) for the determination of soluble zinc content. The amount of zinc solubilized was obtained by subtracting the soluble $\mathrm{Zn}$ of the inoculated sample from the un-inoculated control and expressed as (mg $\mathrm{Zn} / 100 \mathrm{ml}$ ) culture.

\section{Results and Discussion}

Isolation of zinc solubilizing microorganisms from direct sown paddy rhizosphere soils

Rhizospheric soils were collected from different places of Kurnool, Prakasam, Guntur and Anantapur districts such as soils of direct sown paddy growing areas by using quadratic method of soil sample collecting procedure. In Kurnool District (Atmakur, Kothapalle, Jupadu bungalow, Pamulapadu and Velugodu), Prakasam District (Tripuranthakam, Yerragondapalem, Dornala, Markapuram and Giddalur), Guntur District (Vinukonda, Narasaropet, Chilakaluripet, Sattenapalle and Piduguralla) and Anantapur District (Guntakal, Gooty, Pamidi, Tadipatri and Uravakonda) along with particular GPS coordinates for each sampling area was fixed (Table 1) soil samples were collected from each Mandal from different farmer's fields. Coding of the isolates based on the GPS 
location of the collected soil samples (Table 2).

\section{Cultural and morphological characterization}

More number of zinc solubilizing microorganisms was present in the rhizosphere and they are metabolically more active than others. Morphological and cultural characteristics of all the isolates were studied viz., shape, size, margins and colour etc., The morphological features on zinc solubilizing agar plate was studied and they showed small to medium size, dull white or off white, flat, smooth, irregular colonies and there was no pigment production (Table 3 ).

These isolates were found to be gram negative, short stumpy, rod shaped cells when observed under microscope. On the basis of biochemical reactions it was found that Pseudomonas spp. The thirty two isolates were named based on the geographical position of soil sample collected area. Based on microscopic examination and cultural characteristics, Preeti et al., (2011) identified four isolates as Pseudomonas spp. and others as Bacillus spp.

\section{Biochemical characterization}

All the isolates were tested for biochemical characterization viz., Starch hydrolysis, Hydrogen sulphide test, Indole production, Catalase test, Oxidase test, Gelatine liquification, Methyl red test, Vogues Proskauer test, Citrate Utilization, Ammonia production results (Table 2) were revealed that all the zinc solubilizing bacterial isolates were positive for starch hydrolysis expect ZnKAA-4, ZnPYY-1 and ZnGPP-3.

For hydrogen sulphide test all the isolates were positive expect ZnGCC-1, ZnAPP-2.All the isolates were positive for indole production test expect ZnKAA-1, ZnKJJ-4, ZnPTT-3, ZnPYY-2 and ZnAPP-2.For catalase test all isolates are showed positive expect ZnKJJ-2, ZnPMM-3 and ZnGCC-3 are negative. For oxidase test ZnKAA-2, ZnPTT1, ZnPMM-1 and ZnAUU-3 are showed negative remaining all are showed positive.

In the test of gelatine liquefaction all the isolates were positive expect ZnKJJ-1, ZnPYY-1, ZnGCC-2 and ZnGPP-2. For methyl red test ZnKAA-2, ZnKAA-3, ZnPTT-1, ZnPGG-3 and ZnAUU-1 were negative remaining were showed the positive. For the test of vogues proskauer all the isolates were showed the positive expect the ZnKAA-1, ZnKJJ-1, ZnPYY-1, ZnPGG-1 and ZnGPP-1 were showed the negative.

In the test of citrate utilization ZnKJJ-3, ZnPMM-2 and ZnAPP-2 isolates were showed the negative result remaining were all showed the positive result. All the isolates were showed the positive for ammonia production test expect ZnKAA-3, ZnPMM-3 and ZnGCC-3 isolates were showed the negative.

These results are agreed with the Anitha and Kumudini (2014) isolated Pseudomonas from fifteen rhizospheric samples from different regions of India. They characterized morphologically and biochemically and concluded as genus Pseudomonas.

Screening of isolates for their zinc solubilization efficiency

\section{Qualitative method in plat assay}

All the thirty two zinc solubilizing isolates were able to form clear zone of zinc solubilization on zinc solubilizing media plate ranged from $5.12-16.12 \mathrm{~mm}$. Among them ZnKJJ-4 of Pseudomonas spp detected the highest solubilization zone $(16.12 \mathrm{~mm})$ followed by ZnPGG-1 $(15.32 \mathrm{~mm})$ and the lowest solubilization zone was observed with ZnAUU-1 (5.12 mm). 
Table.1 Details of soil samples collected from different districts of Andhra Pradesh

\begin{tabular}{|c|c|c|c|c|c|c|c|c|}
\hline \multirow[t]{2}{*}{ S.No. } & \multirow[t]{2}{*}{ District } & \multirow[t]{2}{*}{ Mandal } & \multirow[t]{2}{*}{ Village } & \multicolumn{2}{|c|}{ Geographical Location } & \multirow[t]{2}{*}{ Sample Code } & \multirow[t]{2}{*}{ Soil type } & \multirow[t]{2}{*}{ Cropping Pattern } \\
\hline & & & & Latitude & Longitude & & & \\
\hline 1. & Kurnool & Atmakur & Atmakur & $15^{0} 91^{\prime} 53^{\prime \prime} \mathrm{N}$ & $78^{0} 70^{\prime} 84^{\prime \prime} \mathrm{E}$ & KAA & Black soil & Rice - Rice \\
\hline 2. & Kurnool & Pamulapadu & Pamulapadu & $15^{0} 81^{\prime} 87^{\prime \prime} \mathrm{N}$ & $78^{0} 50^{\prime} 77^{\prime \prime} \mathrm{E}$ & KPP & Black soil & Rice - Blackgram \\
\hline 3. & Kurnool & Jupadu Bungalow & Jupadu Bungalow & $15^{0} 85^{\prime} 42^{\prime \prime} \mathrm{N}$ & $78^{0} 36^{\prime} 16^{\prime \prime} \mathrm{E}$ & KJJ & Black soil & Rice - Rice \\
\hline 4. & Kurnool & Kothapalle & Kothapalle & $15^{0} 97^{\prime} 84^{\prime \prime} \mathrm{N}$ & $78^{0} 48^{\prime} 63^{\prime \prime} \mathrm{E}$ & KKK & Black soil & Rice - Rice \\
\hline 5. & Kurnool & Velugodu & Velugodu & $15^{0} 72^{\prime} 02^{\prime \prime} \mathrm{N}$ & $78^{0} 57^{\prime} 28^{\prime \prime} \mathrm{E}$ & KVV & Black soil & Rice - Rice \\
\hline 6. & Prakasham & Tripuranthakam & Tripuranthakam & $15^{\circ} 97^{\prime} 80^{\prime \prime} \mathrm{N}$ & $79^{0} 44^{\prime} 61^{\prime \prime} \mathrm{E}$ & PTT & Black soil & Rice - Blackgram \\
\hline 7. & Prakasham & Yerragondapalem & Yerragondapalem & $15^{0} 99^{\prime} 96^{\prime \prime} \mathrm{N}$ & $79^{0} 31^{\prime} 54^{\prime \prime} \mathrm{E}$ & PYY & Black soil & Rice - Rice \\
\hline 8. & Prakasham & Dornala & Dornala & $15^{0} 90^{\prime} 33^{\prime \prime} \mathrm{N}$ & $79^{0} 11^{\prime} 81^{\prime \prime} \mathrm{E}$ & PDD & Black soil & Rice - Rice \\
\hline 9. & Prakasham & Markapuram & Markapuram & $15^{0} 74^{\prime} 28^{\prime \prime} \mathrm{N}$ & $79^{0} 26^{\prime} 77^{\prime \prime} \mathrm{E}$ & PMM & Black soil & Rice - Rice \\
\hline 10. & Prakasham & Giddalur & Giddalur & $15^{0} 37^{\prime} 24^{\prime \prime} \mathrm{N}$ & $78^{0} 95^{\prime} 92^{\prime \prime} \mathrm{E}$ & PGG & Black soil & Rice - Rice \\
\hline 11. & Guntur & Vinukonda & Vinukonda & $16^{0} 04^{\prime} 85^{\prime \prime} \mathrm{N}$ & $79^{0} 75^{\prime} 12^{\prime \prime} \mathrm{E}$ & GVV & Black soil & Rice - Rice \\
\hline 12. & Guntur & Narasaropet & Narasaropet & $16^{0} 23^{\prime} 48^{\prime \prime} \mathrm{N}$ & $80^{\circ} 07^{\prime} 04^{\prime \prime} \mathrm{E}$ & GNN & Black soil & Rice - Blackgram \\
\hline 13. & Guntur & Chilakaluripet & Chilakaluripet & $16^{0} 09^{\prime} 93^{\prime \prime} \mathrm{N}$ & $80^{0} 18^{\prime} 13^{\prime \prime} \mathrm{E}$ & $\mathrm{GCC}$ & Black soil & Rice - Blackgram \\
\hline 14. & Guntur & Sattenapalle & Sattenapalle & $16^{0} 39^{\prime} 92^{\prime \prime} \mathrm{N}$ & $80^{0} 13^{\prime} 70^{\prime \prime} \mathrm{E}$ & GSS & Black soil & Rice - Blackgram \\
\hline 15. & Guntur & Piduguralla & Piduguralla & $16^{0} 47^{\prime} 29^{\prime \prime} \mathrm{N}$ & $79^{0} 90^{\prime} 63^{\prime \prime} \mathrm{E}$ & GPP & Black soil & Rice - Blackgram \\
\hline 16. & Anathapuram & Guntakal & Guntakal & $15^{0} 16^{\prime} 52^{\prime \prime} \mathrm{N}$ & $77^{0} 37^{\prime} 54^{\prime \prime} \mathrm{E}$ & AGG & Black soil & Rice - Rice \\
\hline 17. & Anathapuram & Gooty & Gooty & $15^{0} 11^{\prime} 23^{\prime \prime} \mathrm{N}$ & 77.63'19"E & AGoGo & Black soil & Rice - Rice \\
\hline 18. & Anathapuram & Pamidi & Pamidi & $14^{0} 94^{\prime} 40^{\prime \prime} \mathrm{N}$ & $77^{0} 58^{\prime} 57^{\prime \prime} \mathrm{E}$ & APP & Black soil & Rice - Rice \\
\hline 19. & Anathapuram & Tadipatri & Tadipatri & $14^{0} 91^{\prime} 21^{\prime \prime} \mathrm{N}$ & $78^{0} 00^{\prime} 32^{\prime \prime} \mathrm{E}$ & ATT & Black soil & Rice - Rice \\
\hline 20. & Anathapuramu & Uravakonda & Uravakonda & $14^{0} 94^{\prime} 74^{\prime \prime N}$ & $77^{0} 22^{\prime} 56^{\prime \prime} \mathrm{E}$ & AUU & Black soil & Rice - Rice \\
\hline
\end{tabular}

Table.2 Coding of ZnSB isolates collected from different soil samples according to their geographical position of Andhra Pradesh

\begin{tabular}{|c|c|c|l|}
\hline S.No. & Type of Organism & Number of isolates & \multicolumn{1}{|c|}{ Isolate code } \\
\hline $\mathbf{1 .}$ & ZnSB & 32 & ZnKAA-1, ZnKAA-2, ZnKAA-3, ZnKAA-4, ZnKJJ-1, \\
& & ZnKJJ-2, ZnKJJ-3, ZnKJ-4, ZnPTT-1, ZnPTT-2, \\
& & ZnPTT-3, ZnPTT-4, ZnPYY-1, ZnPYY-2, ZnPMM-1, \\
& & ZnPMM-2, ZnPMM-3, ZnPGG-1, ZnPGG-2, ZnPGG-3, \\
& & ZnGCC-1, ZnGCC-2, ZnGCC-3, ZnGPP-1, ZnGPP-2, \\
& & ZnGPP-3, ZnAPP-1, ZnAPP-2, ZnAPP-3, ZnAUU-1, \\
& & \\
\hline
\end{tabular}


Table.3 Morphological and cultural characterization of Zinc solubilizing bacterial (ZnSB) isolates of different soil samples

\begin{tabular}{|c|c|c|c|c|c|c|c|}
\hline \multirow[t]{2}{*}{ S. No. } & \multirow[t]{2}{*}{ Isolate code } & \multirow[t]{2}{*}{ Gram reaction } & \multirow[t]{2}{*}{ Cell shape } & \multicolumn{4}{|c|}{ Colony morphology } \\
\hline & & & & Colour & Form & Elevation & Margin \\
\hline 1. & ZnKAA-1 & -ve & Rod & White & Irregular & Raised & Undulate \\
\hline 2. & ZnKAA-2 & -ve & Rod & Creamy white & Circular & Flat & Entire \\
\hline 3. & ZnKAA-3 & -ve & Rod & Creamy white & Irregular & Umbonate & Lobate \\
\hline 4. & ZnKAA-4 & -ve & Rod & Yellowish white & Irregular & Convex & Curled \\
\hline 5. & ZnKJJ-1 & -ve & Rod & Creamy white & Irregular & Raised & Undulate \\
\hline 6. & ZnKJJ-2 & -ve & Rod & White & Circular & Convex & Entire \\
\hline 7. & ZnKJJ-3 & -ve & Rod & White & Circular & Flat & Curled \\
\hline 8. & ZnKJJ-4 & -ve & Rod & White & Irregular & Flat & Entire \\
\hline 9. & ZnPTT-1 & -ve & Rod & White & Irregular & Convex & Entire \\
\hline 10. & ZnPTT-2 & -ve & Rod & Off White & Irregular & Umbonate & Curled \\
\hline 11. & ZnPTT-3 & -ve & Rod & Creamy white & Circular & Convex & Entire \\
\hline 12. & ZnPTT-4 & -ve & Rod & White & Circular & Raised & Undulate \\
\hline 13. & ZnPYY-1 & -ve & Rod & White & Irregular & Convex & Curled \\
\hline 14. & ZnPYY-2 & -ve & Rod & White & Irregular & Flat & Undulate \\
\hline 15. & ZnPMM-1 & -ve & Rod & White & Irregular & Crateriform & Filiform \\
\hline 16. & ZnPMM-2 & -ve & Rod & Light Yellow & Circular & Flat & Entire \\
\hline 17. & ZnPMM-3 & -ve & Rod & Whitish Yellow & Irregular & Flat & Curled \\
\hline 18. & ZnPGG-1 & -ve & Rod & White & Irregular & Umbonate & Undulate \\
\hline 19. & ZnPGG-2 & -ve & Rod & White & Irregular & Convex & Entire \\
\hline 20. & ZnPGG-3 & -ve & Rod & White & Irregular & Raised & Lobate \\
\hline 21. & ZnGCC-1 & -ve & Rod & White & Irregular & Convex & Lobate \\
\hline 22. & ZnGCC-2 & -ve & Rod & White & Circular & Flat & Entire \\
\hline 23. & $\mathrm{ZnGCC}-3$ & -ve & Rod & White & Irregular & Raised & Entire \\
\hline 24. & ZnGPP-1 & -ve & Rod & White & Filamentous & Flat & Lobate \\
\hline 25. & ZnGPP-2 & -ve & Rod & Dark Yellowish & Circular & Convex & Curled \\
\hline 26. & ZnGPP-3 & -ve & Rod & White & Filamentous & Umbonate & Curled \\
\hline 27. & ZnAPP-1 & -ve & Rod & White & Irregular & Raised & Curled \\
\hline 28. & ZnAPP-2 & -ve & Rod & White & Irregular & Convex & Undulate \\
\hline 29. & ZnAPP-3 & -ve & Rod & Light green & Circular & Umbonate & Entire \\
\hline 30. & ZnAUU-1 & -ve & Rod & White & Circular & Raised & Curled \\
\hline 31. & ZnAUU-2 & -ve & Rod & Yellow & Irregular & Convex & Undulate \\
\hline 32. & ZnAUU-3 & -ve & Rod & Milky white & Circular & Flat & Entire \\
\hline
\end{tabular}


Table.4 Estimation of Zinc solubilization quantitatively and qualitatively for the screening of $\mathrm{ZnSB}$ isolates from different soil samples

\begin{tabular}{|c|c|c|c|c|c|}
\hline \multirow{3}{*}{ S.No. } & \multirow{3}{*}{$\begin{array}{l}\text { Isolate } \\
\text { name }\end{array}$} & \multirow{3}{*}{$\begin{array}{l}\text { Soluble Zn } \\
\text { concentration } \\
\text { ug ml }^{-1}\end{array}$} & \multicolumn{3}{|c|}{ Zinc solubilisation } \\
\hline & & & \multirow{2}{*}{$\begin{array}{c}\text { Zinc } \\
\text { solubilization } \\
\text { index (ZSI) }\end{array}$} & \multicolumn{2}{|c|}{ Zone diameter (mm) } \\
\hline & & & & $\begin{array}{c}\text { Solubilization } \\
\text { zone }\end{array}$ & $\begin{array}{c}\text { Culture } \\
\text { diameter }\end{array}$ \\
\hline 1. & ZnKAA-1 & 1.51 & 3.85 & 9.12 & 3.20 \\
\hline 2. & ZnKAA-2 & 1.45 & 3.03 & 8.57 & 4.23 \\
\hline 3. & ZnKAA-3 & 1.75 & 3.37 & 5.70 & 2.41 \\
\hline 4. & ZnKAA-4 & 2.45 & 4.03 & 6.12 & 2.02 \\
\hline 5. & ZnKJJ-1 & 1.54 & 3.70 & 9.22 & 3.41 \\
\hline 6. & ZnKJJ-2 & 2.03 & 4.29 & 10.21 & 3.10 \\
\hline 7. & ZnKJJ-3 & 1.61 & 3.00 & 6.00 & 3.00 \\
\hline 8. & ZnKJJ-4 & 2.89 & 4.91 & 16.12 & 4.12 \\
\hline 9. & ZnPTT-1 & 1.32 & 2.95 & 8.00 & 4.10 \\
\hline 10. & ZnPTT-2 & 1.45 & 2.73 & 7.13 & 4.12 \\
\hline 11. & ZnPTT-3 & 1.84 & 3.33 & 10.15 & 4.35 \\
\hline 12. & ZnPTT-4 & 1.36 & 3.26 & 7.10 & 3.14 \\
\hline 13. & ZnPYY-1 & 1.96 & 3.85 & 12.01 & 4.21 \\
\hline 14. & ZnPYY-2 & 1.98 & 3.90 & 9.14 & 3.15 \\
\hline 15. & ZnPMM-1 & 1.41 & 3.43 & 8.30 & 3.41 \\
\hline 16. & ZnPMM-2 & 1.24 & 3.28 & 8.22 & 3.60 \\
\hline 17. & ZnPMM-3 & 1.21 & 3.25 & 9.21 & 4.10 \\
\hline 18. & ZnPGG-1 & 2.85 & 4.46 & 15.32 & 4.43 \\
\hline 19. & ZnPGG-2 & 1.79 & 3.99 & 9.01 & 3.01 \\
\hline 20. & ZnPGG-3 & 1.45 & 3.36 & 8.05 & 3.41 \\
\hline 21. & ZnGCC-1 & 1.22 & 2.50 & 6.20 & 4.12 \\
\hline 22. & ZnGCC-2 & 2.32 & 4.07 & 9.52 & 3.10 \\
\hline 23. & ZnGCC-3 & 1.78 & 3.92 & 12.01 & 4.11 \\
\hline 24. & ZnGPP-1 & 1.32 & 3.43 & 7.64 & 3.14 \\
\hline 25. & ZnGPP-2 & 1.13 & 2.37 & 5.48 & 4.01 \\
\hline 26. & ZnGPP-3 & 1.96 & 3.98 & 9.31 & 3.12 \\
\hline 27. & ZnAPP-1 & 1.89 & 3.43 & 10.21 & 4.21 \\
\hline 28. & ZnAPP-2 & 1.42 & 3.35 & 9.41 & 4.01 \\
\hline 29. & ZnAPP-3 & 1.65 & 3.70 & 8.12 & 3.01 \\
\hline 30. & ZnAUU-1 & 1.01 & 2.22 & 5.12 & 4.21 \\
\hline 31. & ZnAUU-2 & 1.48 & 3.73 & 9.44 & 3.46 \\
\hline 32. & ZnAUU-3 & 1.26 & 3.58 & 8.50 & 3.30 \\
\hline SE(m) & & 0.04 & & 0.06 & \\
\hline $\mathrm{CD}(\mathrm{P}=0.05)$ & & 0.09 & & 0.14 & \\
\hline CV & & 1.84 & & 1.43 & \\
\hline
\end{tabular}


Table.5 Biochemical and physiological characterization of Zinc Solubilizing Bacterial $(\mathrm{ZnSB})$ isolates collected from different soil samples

\begin{tabular}{|c|c|c|c|c|c|c|c|c|c|c|c|}
\hline S.No. & Isolate code & $\begin{array}{c}\text { Starch } \\
\text { hydrolysis }\end{array}$ & $\begin{array}{c}\text { Hydrogen } \\
\text { sulphide test }\end{array}$ & $\begin{array}{c}\text { Indole } \\
\text { production }\end{array}$ & $\begin{array}{c}\text { Catalase } \\
\text { test }\end{array}$ & $\begin{array}{c}\text { Oxidase } \\
\text { test }\end{array}$ & $\begin{array}{c}\text { Gelatine } \\
\text { liquification }\end{array}$ & $\begin{array}{l}\text { Methyl } \\
\text { red test }\end{array}$ & $\begin{array}{c}\text { Vogues } \\
\text { Proskauer test }\end{array}$ & $\begin{array}{c}\text { Citrate } \\
\text { Utilization }\end{array}$ & $\begin{array}{c}\text { Ammonia } \\
\text { production }\end{array}$ \\
\hline 1. & ZnKAA-1 & + & + & - & + & + & + & + & - & + & + \\
\hline 2. & ZnKAA-2 & + & + & + & + & - & + & - & + & + & + \\
\hline 3. & ZnKAA-3 & + & + & + & + & + & + & - & + & + & - \\
\hline 4. & ZnKAA-4 & - & + & + & + & + & + & + & + & + & + \\
\hline 5. & ZnKJJ-1 & + & + & + & + & + & - & + & - & + & + \\
\hline 6. & ZnKJJ-2 & + & + & + & - & + & + & + & + & + & + \\
\hline 7. & ZnKJJ-3 & + & + & + & + & + & + & + & + & - & + \\
\hline 8. & ZnKJJ-4 & + & + & - & + & + & + & + & + & + & + \\
\hline 9. & ZnPTT-1 & + & + & + & + & - & + & - & + & + & + \\
\hline 10. & ZnPTT-2 & + & + & + & + & + & + & + & + & + & + \\
\hline 11. & ZnPTT-3 & + & + & - & + & + & + & + & + & + & + \\
\hline 12. & ZnPTT-4 & + & + & + & + & + & + & + & + & + & + \\
\hline 13. & ZnPYY-1 & - & + & + & + & + & - & + & - & + & + \\
\hline 14. & ZnPYY-2 & + & + & - & + & + & + & + & + & + & + \\
\hline 15. & ZnPMM-1 & + & + & + & + & - & + & + & + & + & + \\
\hline 16. & ZnPMM-2 & + & + & + & + & + & + & + & + & - & + \\
\hline 17. & ZnPMM-3 & + & + & + & - & + & + & + & + & + & - \\
\hline 18. & ZnPGG-1 & + & + & + & + & + & + & + & - & + & + \\
\hline 19. & ZnPGG-2 & + & + & + & + & + & + & + & + & + & + \\
\hline 20. & ZnPGG-3 & + & + & + & + & + & + & - & + & + & + \\
\hline 21. & ZnGCC-1 & + & - & + & + & + & + & + & + & + & + \\
\hline 22. & ZnGCC-2 & + & + & + & + & + & - & + & + & + & + \\
\hline 23. & ZnGCC-3 & + & + & + & - & + & + & + & + & + & - \\
\hline 24. & ZnGPP-1 & + & + & + & + & + & + & + & - & + & + \\
\hline 25. & ZnGPP-2 & + & + & + & + & + & - & + & + & + & + \\
\hline 26. & ZnGPP-3 & - & + & + & + & + & + & + & + & + & + \\
\hline 27. & ZnAPP-1 & + & + & + & + & + & + & + & + & + & + \\
\hline 28. & ZnAPP-2 & + & - & - & + & + & + & + & + & - & + \\
\hline 29. & ZnAPP-3 & + & + & + & + & + & + & + & + & + & + \\
\hline 30. & ZnAUU-1 & + & + & + & + & + & + & - & + & + & + \\
\hline 31. & ZnAUU-2 & + & + & + & + & + & + & + & + & + & + \\
\hline 32. & ZnAUU-3 & + & + & + & + & - & + & + & + & + & + \\
\hline
\end{tabular}


Plate.1 Zinc solubilization of different bacterial isolates on $\mathrm{ZnSB}$ medium
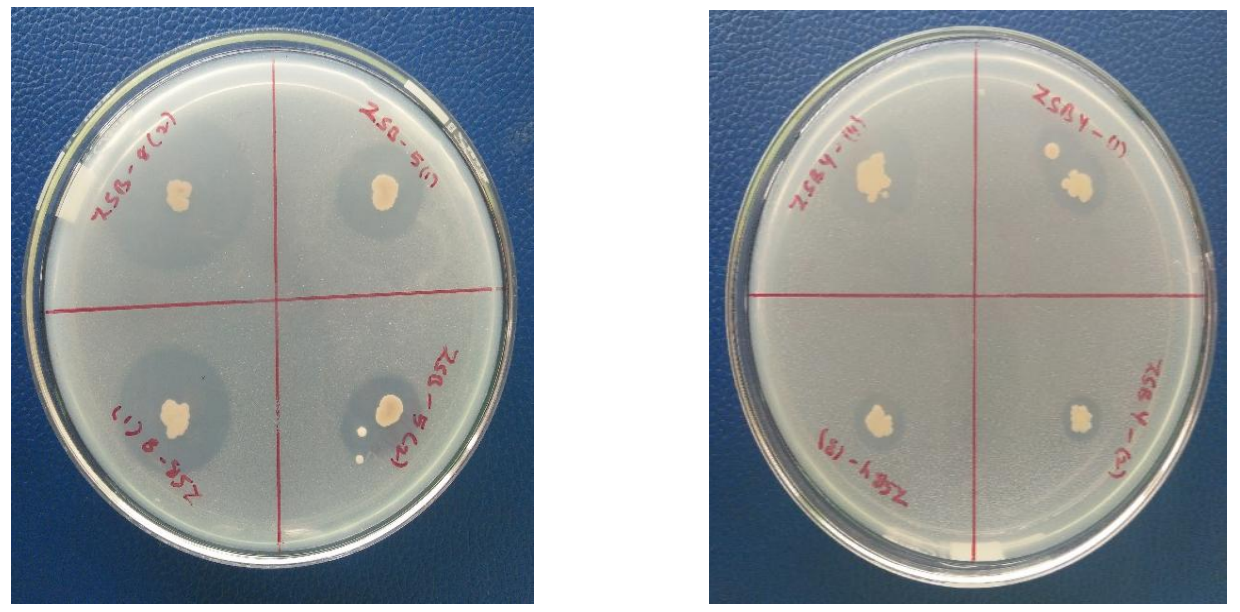

Plate.2 Zinc solubilization byZnKJJ-4bacterial isolate
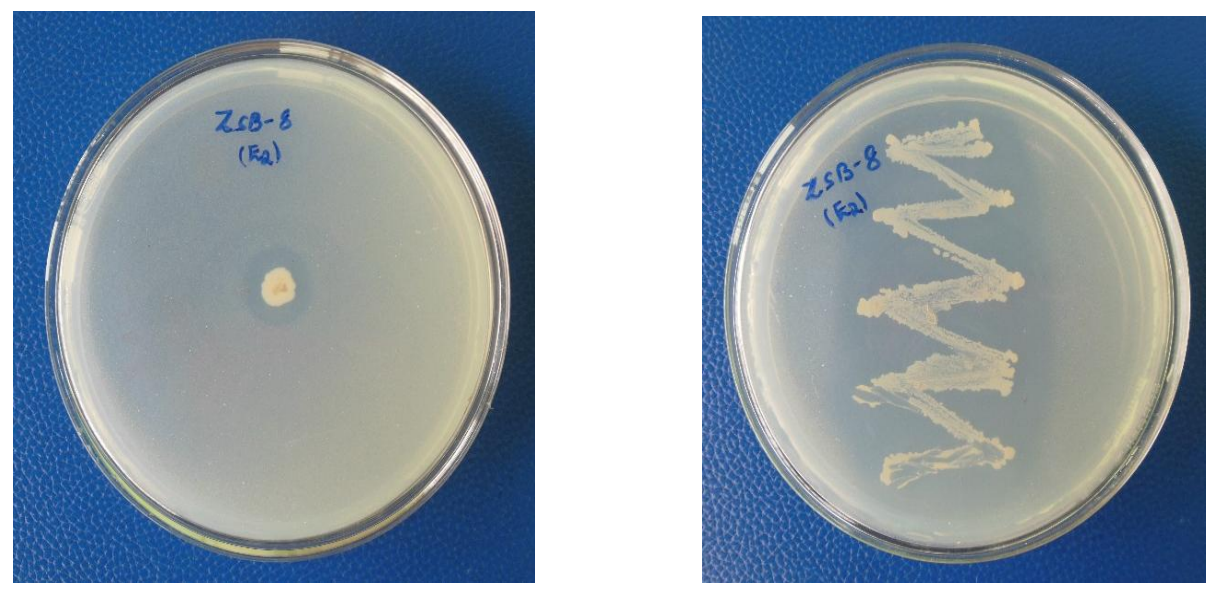

Plate.3 Zinc solubilization by ZnAUU-1bacterial isolate
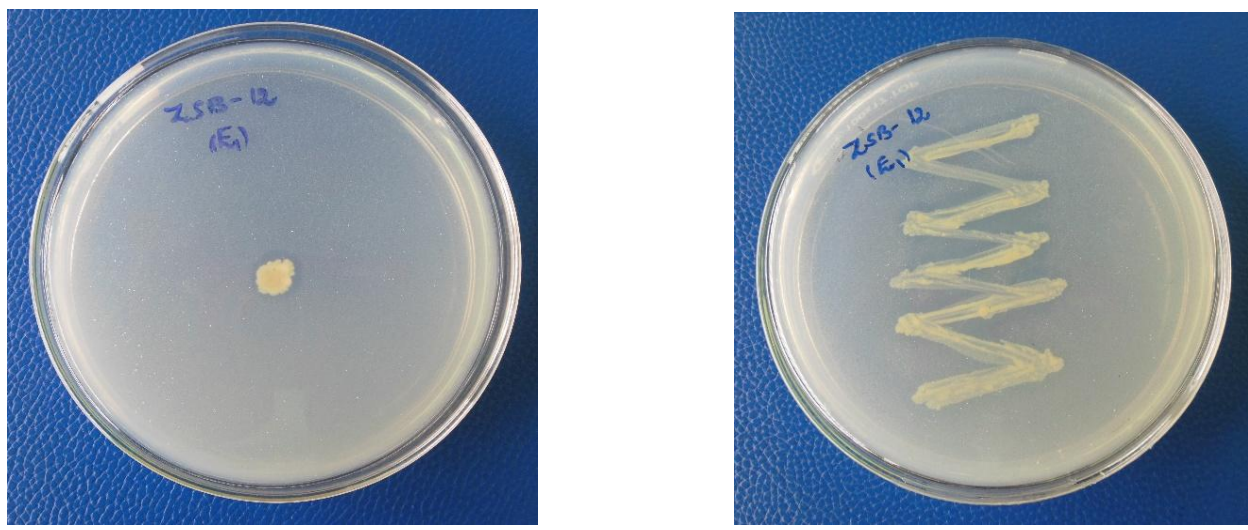
Plate.4 Different biochemical tests for characterization of $\mathrm{ZnSB}$ isolates

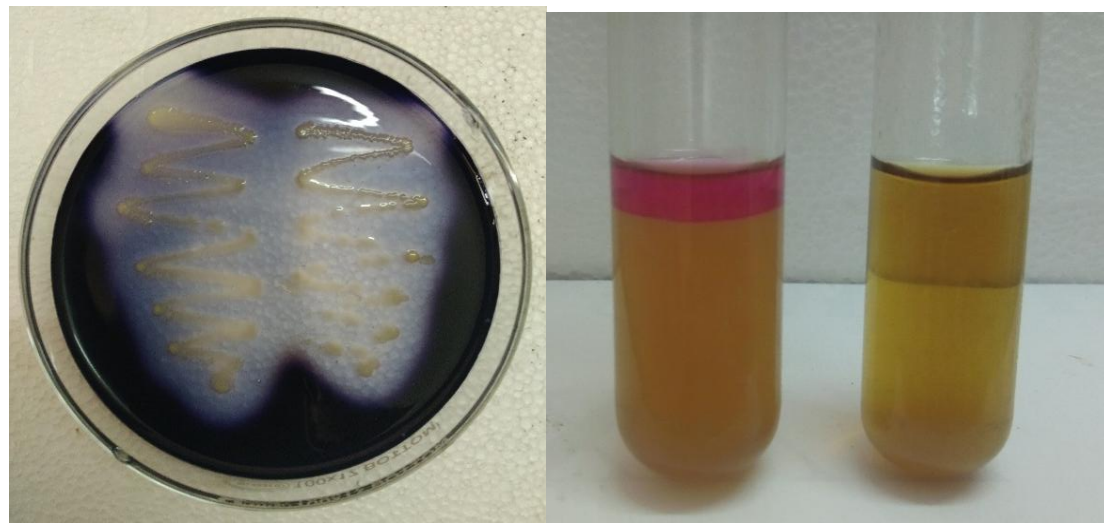

Starch hydrolysis test

Vogues Proskauer test

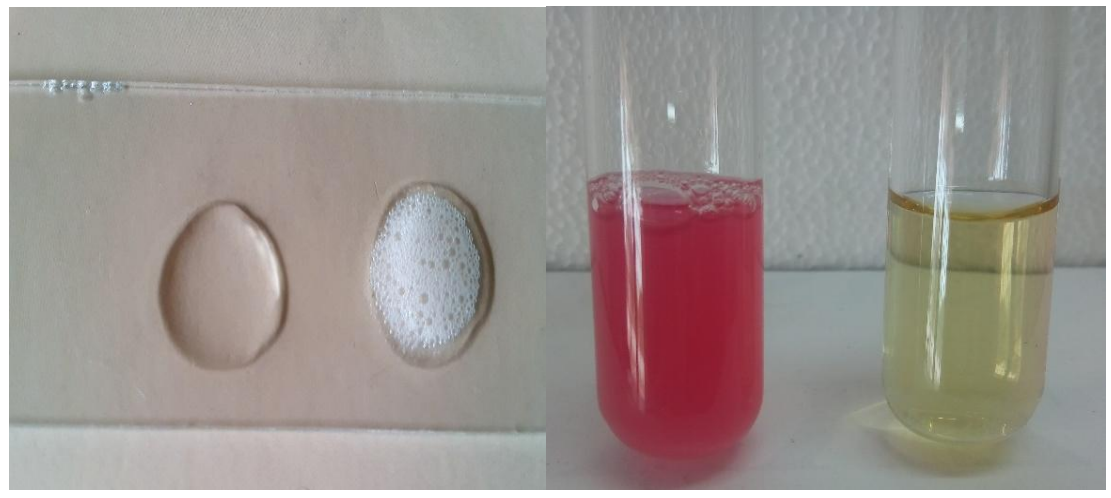

Catalase test

Methyl red test

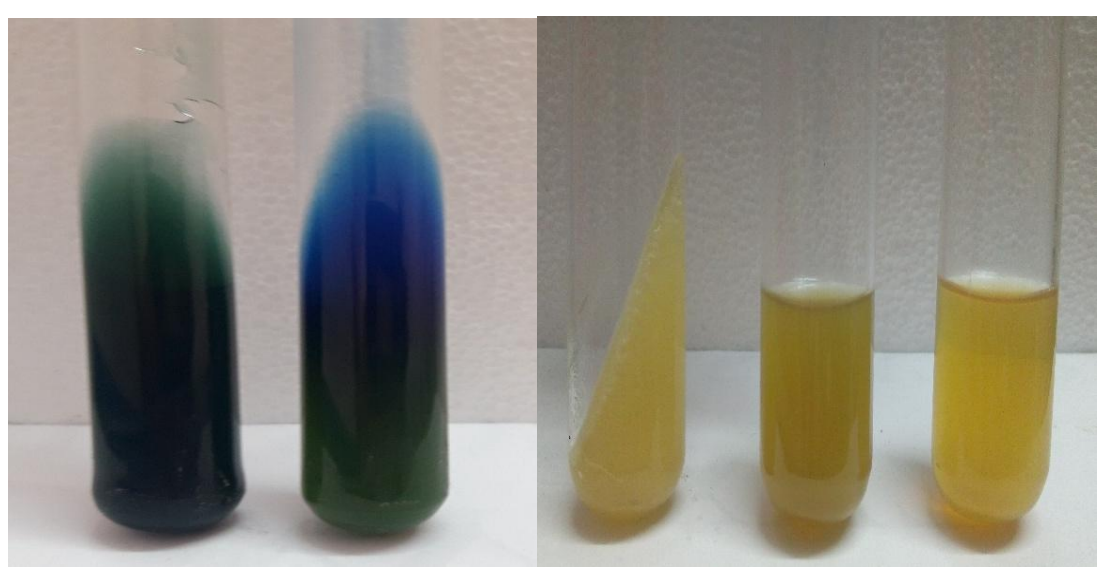

Citrate test

Gelatine test 
Zinc solubulization index were also highest in ZnKJJ-4 (4.91) followed by ZnPGG-1 (4.46)and the lowest solubilization index was observed in ZnAUU-1 (2.22). This results were correlated with the Tensingh et al., (2015) identified the selected strains were Bacillus and Pseudomonas. The isolated strains were characterized under in vitro conditions. They showed solubilization zone ranges from $2-5 \mathrm{~mm}$ at $28-30^{\circ} \mathrm{C}$. The highest solubilization was observed with Pseudomonas putida $(5 \mathrm{~mm})$ followed by Pseudomonas flourescens (4 $\mathrm{mm})$ and the lowest solubilization was observed in Bacillus megaterium $(2 \mathrm{~mm})$.

\section{Quantitative method in broth assay}

All the thirty two zinc solubilizing isolates were able to solubilize the available zinc in zinc solubilizing broth with mineral salts medium supplemented with insoluble source of zinc phosphate at $0.1 \%$ concentration. Among them ZnKJJ-4 recorded the more available zinc content of $2.89 \mathrm{ug} \mathrm{ml}^{-1}$. Second best was showed by ZnPGG-1 i.e., $2.85 \mathrm{ug} \mathrm{ml}^{-}$ ${ }^{1}$.The lowest was shown by $\mathrm{Zn}$ AUU-1 with $1.01 \mathrm{ug} \mathrm{ml}^{-1}$.Similar results were observed by Kajal and Desai (2015) isolated zinc solubilizing bacteria from alkaline fields. Among 309 isolates, 141 isolates were positive for zinc solubilization. Considering the quantitative aspects of solubilization efficiency the bacterial isolates ranged from (116-366\%) on $0.1 \%$ zinc oxide incorporated medium. Based on solubilization efficiency five isolates with highest $\mathrm{Zn}$ solubilization efficiency were chosen for further tests related to multiple plant growth promoting traits.

In conclusion, zinc available in soil in various forms it was solubilized by the certain microorganisms known as zinc solubilizing microorganisms. Pseudomonas spp reported to be active in the zinc solubilization process.
Initially zinc solubilizing bacteria was isolated and grown in respective media with insoluble source of zinc phosphate. All the isolates were characterized morphologically and biochemically. The efficient isolates were subjected to further characterization. The isolates were selected based on their good performance of PGPR characters. For Zinc solubilisation the individual isolates were able to form a solubilization zone ranged from 5.12 - $16.12 \mathrm{~mm}$. The highest zinc solubilization index (4.91) was recorded in ZnKJJ-4 and lowest zinc solubilization index was observed in ZnAUU-1 (2.22).

\section{References}

Abaid-Ullah M., Hassan M. N., Jamil M., Brader G., Shah M. K. N., Sessitsch A., et al. (2015). Plant growth promoting rhizobacteria: an alternate way to improve yield and quality of wheat (Triticum aestivum). International Journal of Agricultural Biology. 17: 5160.

Anitha, G and Kumudini, B.S. 2014. Isolation and characterization of fluorescent pseudomonads and their effect on plant growth promotion. Journal of Environmental Biology. 35: 627-634.

Barthalomew, J.W and Mittewer, T. 1950. A simplified bacterial strain. Stain Technology. 25: 152-153.

Beishir, L. 1991. Microbiology in Practice: A Self instructional laboratory course. $5^{\text {th }}$ ed. Harper Collins College Publishers: New York. Pp. 1-3.

Collins. C.H and Lyne, P.M. 1970. Microbiological methods. London: Butter Worths. 36: 463-468.

Crown, S.T and Gen, J. 1998. Micro method for the methyl red test. Microbiology. 9: 101-109.

FAO. $2017 . \quad$ FAOSTAT. www.fao.org/nr/water/aquastat/data/quer y/index. 
Fasim, F., Ahmed, N., Parsons, R and Gadd, G.M. 2002. Solubilization of zinc salts by bacterium isolated from the air environment of tannery. FEMS Microbiology Letters. 213: 1-6.

Isenberg, H.D and Sundheim, L.H. 1958. Indole reactions in bacteria. Journal of Bacteriology. 75: 682-690.

Juanda, J.I.H. 2005. Screening of soil bacteria for plant growth promoting activities in vitro.The Journal of Agricultural Science. 4: $27-31$.

MacFaddin, J.F. 2000. Biochemical tests for identification of medical bacteria, $3^{\text {rd }}$ ed. Lippincott Williams and Wilkins, Philadelphia, PA. 6 (1): 503-642.

Pawar A., Ismail S., Mundhe S and Patil V. D. 2015. Solubilization of insoluble zinc compounds by different microbial isolates in vitro condition. International Journal of Tropical Agriculture. 33. 865869.

Preeti, T., Ekka, S.R and Tripathi, R. 2011. In Vitro Study of Pseudomonas spp. isolated from Soil. Journal of Phytology. 3 (4): 21-23.

Rangaswami, G and Bagayaraj, D.J. 1993. Microbial biotechnology. In: Agricultural micro biology. Prentice Hall of India Private Limited. New Delhi. Pp. 389-405.

Rodriguiz, H., Gonzalez, T., Goire, I and Bashan, Y. 2004. Gluconic acid production and phosphate solubilization by the plant growth promoting bacterium Azospirillum spp. Nature. 91: 552-555.

Saravanan V. S., Madhaiyan M and Thangaraju M. 2007. Solubilization of zinc compounds by the diazotrophic, plant growth promoting bacterium Gluconacetobacter diazotrophicus. Chemosphere. 66: 1794-1798.

Thompson, J. P. 1996. Correction of dual phosphorous and zinc deficiencies of linseed with cultivars of vesiculararbuscular mycorrhizal fungi. Soil Biology and Biochemistry. 28: 941-951.

\section{How to cite this article:}

Vinod Babu, S., A. Vijaya Gopal, N. Trimurtulu, G. Kishore Babu and Sree Lakshmi, B. 2020. Isolation, Screening and Characterization of Zinc Solubilizing Microorganisms from Direct Sown Paddy (Oryza sativa L.) Rhizospheric Soils of Andhra Pradesh Int.J.Curr.Microbiol.App.Sci. 9(09): 3334-3346. doi: https://doi.org/10.20546/ijcmas.2020.909.415 